\title{
Evaluation the antinociceptive and anti- inflammatory effect, of new rigid, propoxy benzopyrane-3,4 Di-hydroxychalcone derivative by Hot-plate, Formaline and Plethysmography
}

Payam Khazaeli ${ }^{1,4^{*}}$, Mamoud Reza Heidari ${ }^{2,4}$, Alireza Foroumadi ${ }^{3,4}$, Mehdi Kalati ${ }^{4,5}$

From $1^{\text {st }}$ International Congress on Neurobiology and Clinical Psychopharmacology and European

Psychiatric Association Conference on Treatment Guidance

Thessaloniki, Greece. 19-22 November 2009

\section{Background}

There are many reports indicating the analgesic and anti inflammatory effects of 3,4-dihydroxy chalcones. In this study antinociceptive and anti-inflammator effects of rigid derivative 3-(3,4-dihydroxybenzylidene)-7-propoxy benzopyran-4-one, were evaluated by Formalin, Hot plate and Carageenan tests.

\section{Materials and methods}

Experimental doses of 50, 75 and $100 \mathrm{mg} / \mathrm{kg}$ of 3,4DHC were injected to mice and the analgesic and anti inflammatory effects evaluated by Formalin, Hotplate and Carageenan tests. Effective dose compared with Morphine and Ibuprofen.

\section{Results}

The result showed that, propoxy chalcone with dose of $75 \mathrm{mg} / \mathrm{kg}$ induced significant anti nociception and anti inflammation in Formalin and Carageenan tests. The results showed that the dose of $75 \mathrm{mg} / \mathrm{kg}$ of 3,4-DHC induces significant analgesia in 45 and 60 minutes in hot plate test .The analgesic effect of the most effective dose of 3,4-Dihydroxy chalcone $75 \mathrm{mg} / \mathrm{kg}$ was lower than morphine $(2.5 \mathrm{mg} / \mathrm{kg})$ in all time in Formalin and Hot plate tests. The analgesic effect of DHC was higher than Ibuprofen $(200 \mathrm{mg} / \mathrm{kg})$ in $0-5$ minute in Formalin test and in 45 and 60 minutes in Hot plate test, but in chronic phase of Formalin test was nearly equal to Ibuprofen. In Carageenan test, the anti inflammatory effect of 3,4-DHC was higher than Ibuprofen $(200 \mathrm{mg} / \mathrm{kg}$ ) and morphine $(2.5 \mathrm{mg} / \mathrm{kg})$ in the first and third hours. Therefore it seems that 3,4-DHC has better anti-inflammatory effect rather than analgesic effect. The doses of 75 and $100 \mathrm{mg} / \mathrm{kg}$, induced lethargy in mice.

\section{Conclusions}

The results showed that the modification of this structure of DHC, may lead to more effective derivative with significant analgesic effect and it could be used for more studies to access a clinical use of 3,4-DHC as a drug.

\section{Author details \\ ${ }^{1}$ Department of Pharmaceutics, Faculty of Pharmacy, Pharmaceutics Research Center, Kerman University of Medical Sciences, Kerman, Iran. ${ }^{2}$ Department of Toxicology and Pharmacology, Faculty of Pharmacy, Pharmaceutics and Physiology Research Centers, Kerman University of Medical Sciences, Kerman, Iran. ${ }^{3}$ Department of Medicinal Chemistry, Faculty of Pharmacy and Pharmaceutical Sciences Research Center, Tehran University of Medical Sciences, Tehran, Iran. ${ }^{4}$ Department of Cardiology, Faculty of Medicine, Kerman University of Medical Sciences, Kerman, Iran. ${ }^{5}$ Kerman Neuroscience Research Center, Kerman University of Medical Sciences, Kerman, Iran.}

Published: 22 April 2010

doi:10.1186/1744-859X-9-S1-S136

Cite this article as: Khazaeli et al:: Evaluation the antinociceptive and anti-inflammatory effect, of new rigid, propoxy benzopyrane-3,4 Di-hydroxychalcone derivative by Hot-plate, Formaline and Plethysmography. Annals of General Psychiatry 2010 9(Suppl 1):S136. 